\title{
Relationship between Choroidal Thickness and Visual Field Impairment in Acute Zonal Occult Outer Retinopathy
}

\author{
Yuki Hashimoto, ${ }^{1}$ Wataru Saito, ${ }^{1,2}$ Michiyuki Saito, ${ }^{1}$ Yuka Hasegawa, ${ }^{1}$ Akari Takita, ${ }^{1}$ \\ Shohei Mori, ${ }^{1}$ Kousuke Noda, ${ }^{1}$ and Susumu Ishida ${ }^{1}$ \\ ${ }^{1}$ Department of Ophthalmology, Faculty of Medicine and Graduate School of Medicine, Hokkaido University, Sapporo, Japan \\ ${ }^{2}$ Kaimeido Eye and Dental Clinic, Sapporo, Japan \\ Correspondence should be addressed to Wataru Saito; wsaito@med.hokudai.ac.jp
}

Received 8 March 2017; Accepted 29 May 2017; Published 5 July 2017

Academic Editor: Toshihide Kurihara

Copyright (C) 2017 Yuki Hashimoto et al. This is an open access article distributed under the Creative Commons Attribution License, which permits unrestricted use, distribution, and reproduction in any medium, provided the original work is properly cited.

\begin{abstract}
Purpose. To evaluate sequential changes in choroidal thickness at the affected area in patients with acute zonal occult outer retinopathy (AZOOR). Methods. This retrospective observational case series included 14 affected eyes and 6 unaffected fellow eyes from 10 AZOOR patients with impaired macular area. Using enhanced depth imaging optical coherence tomography, choroidal thicknesses at the subfovea and at nasal and temporal sites $1000 \mu \mathrm{m}$ away from the fovea were manually measured at baseline and 3 and 6 months thereafter. Changes in the choroidal thicknesses and the average threshold at the affected area on Humphrey perimetry were compared during the 6-month follow-up. Results. In AZOOR eyes, the average threshold at the affected area significantly increased over time, while outer retinal structure ameliorated. The mean choroidal thicknesses at all the sites measured significantly decreased at 3 and 6 months compared with baseline values in AZOOR eyes, but not in fellow eyes. There was an inverse correlation between the changing rates of the average threshold and the subfoveal choroidal thickness at 6 months from baseline. Conclusion. The current data suggest that choroidal thickness at AZOOR-affected area significantly decreased with regression of AZOOR and this anatomical change correlated with the functional recovery.
\end{abstract}

\section{Introduction}

Acute zonal occult outer retinopathy (AZOOR), first described by Gass in 1992, is an idiopathic syndrome with acute outer retinal impairment $[1,2]$. Visual field defects in AZOOR patients are caused by outer retinal impairment and are demonstrated by decreased responses on multifocal electroretinography (ERG) [3] and disrupted ellipsoid zones detected by spectral domain optical coherence tomography (SD-OCT) $[4,5]$. Fundus autofluorescence can also visualize retinal pigment epithelium (RPE) abnormalities in the affected area during the acute and chronic stages of AZOOR $[6,7]$.

The pathogenesis causing the photoreceptor impairment in AZOOR is still unresolved. Regarding the association between AZOOR and the choroid, abnormalities on indocyanine green angiography (ICGA) have been reported as hypofluorescence in areas related or unrelated to the lesions [8-13]. Moreover, we recently demonstrated using laser speckle flowgraphy (LSFG) that choroidal blood flow velocity at AZOOR lesions significantly increased as visual function and outer retinal structure improved [8]. Since the choriocapillaris supplies nutrients and oxygen to the photoreceptors, these observations suggest a correlation between choroidal circulatory impairment and the pathogenesis of AZOOR, although further studies are needed to verify the relationship [14].

There are only a few reports that evaluated choroidal thickness in AZOOR. Subfoveal choroidal thickness in AZOOR patients tended to be less than that in normal eyes without retinal diseases $(243 \mu \mathrm{m}$ versus $289 \mu \mathrm{m})$ [6], although there was no significant difference between the two groups. The tendency may have resulted from the higher frequency of myopia among AZOOR patients. In 4 patients 
with AZOOR associated with punctuate inner choroidopathy (PIC), central choroidal thickness decreased together with the recovery of outer retinal structure and visual function [15]. In the acute stage of PIC, however, increased choroid thickness was noted at the affected area, even when it was not complicated by AZOOR [16]. By contrast, it remains unknown how choroidal thickness alters during the clinical course of AZOOR.

The aim of this study was to evaluate sequential changes in choroidal thickness at the affected area in eyes with AZOOR.

\section{Methods}

2.1. Patients. This retrospective observational case series included 14 affected eyes and 6 unaffected fellow eyes as controls from 10 patients with AZOOR (2 men and 8 women) who visited Hokkaido University Hospital from May 2011 through September 2014. Inclusion criteria were AZOOR eyes showing outer retinal morphological abnormalities in the macular area on OCT at the initial visit, regardless of the types of scotomata, and receiving choroidal thickness measurements with enhanced depth imaging- (EDI-) OCT for up to 6 months after baseline. The current study was approved by the ethics committee of Hokkaido University Hospital and followed the tenets of the Declaration of Helsinki. Informed consent was obtained from each subject after the nature and potential consequences of the study had been explained.

2.2. Diagnosis. The diagnostic criteria for AZOOR were as follows: acute visual field or vision loss usually with concurrent photopsia; one or more visual field defect regions that could not be explained by funduscopic examination or fluorescein angiography (FA); decreased multifocal ERG responses corresponding to retinal sites with visual field defects; and outer retinal morphologic abnormalities, including absence or discontinuity of the ellipsoid zone and/or the interdigitation zone on OCT $[2,4,5,17]$. Patients who showed funduscopic and angiographic findings consistent with white dots observed in AZOOR complex such as multiple evanescent white dot syndrome (MEWDS) were excluded.

2.3. Treatment. Of the 10 patients included in this study, 6 patients (9 eyes) who had the best-corrected visual acuity (BCVA) of more than 0.5 at the initial visit with nonprogressive clinical courses or who refused the administration of systemic corticosteroids despite an initially worsened BCVA were followed up without treatment (cases 1-6). Four patients (5 eyes) with an initially worsened BCVA and progressive central or paracentral visual function loss were treated with systemic corticosteroids, including corticosteroid pulse therapy or oral prednisolone (cases 7-10). The regimen for corticosteroid pulse therapy has been described previously [8]. In cases with worsening visual field defects on perimetry or worsening subjective symptoms during follow-up visits, the dose was temporarily increased.
2.4. Ophthalmic Examinations. At the initial visit, each patient underwent thorough ophthalmic examinations including BCVA, indirect ophthalmoscopy, FA, ICGA, $20 \mathrm{~J}$ single-flash ERG, and SD-OCT (cross-sectional retinal Bscans of $5 \times 5$ lines) combined with EDI-OCT (RS-3000 Advance; NIDEK, Gamagori, Japan). Several days later, these examinations were followed by visual field testing (Goldmann perimetry and a Humphrey 30-2 Swedish Interactive Threshold Algorithm (SITA) standard test), fundus autofluorescence, and multifocal ERG. BCVA, Humphrey perimetry, and OCT findings were assessed at baseline as well as at 3 and 6 months after baseline.

2.5. EDI-OCT. EDI-OCT measurements were obtained for each of the evaluation points at the initial visit and 3 and 6 months after the initial visit for nontreated AZOOR patients and before treatment and 3 and 6 months after the start of treatment for AZOOR patients receiving systemic corticosteroids. Using a horizontal scan through the fovea (scan length, $9.0 \mathrm{~mm}$ ), choroidal thicknesses at the subfovea and at nasal and temporal sites $1000 \mu \mathrm{m}$ away from the subfovea were determined by manually measuring the distance from the outer border of the hyperreflective line corresponding to the RPE to the outer border of the choroid in 14 AZOOR eyes and 6 unaffected fellow eyes (Figures 1(e) and 1(f)). Three authors (Yuki H., Yuka H., and A.T.), who were blinded to the subjects' clinical information, independently examined EDI-OCT images. We assessed the statistical significance of differences in average choroidal thickness values between stages.

2.6. Visual Function. Using Humphrey perimetry, 4 adjacent threshold points at the lesion area were selected at the lesion area in the AZOOR eyes (Figures 1(c) and 1(d); red squares) and the corresponding area to the 4 points evaluated for each affected eye in the fellow eyes. The average threshold was calculated as the numerical index of changes in visual field impairment.

2.7. Statistics. All results are expressed as the mean \pm standard deviation (SD). BCVA was converted to the logarithm of the minimum angle of resolution (logMAR) scale for the purpose of statistical analyses. Mann-Whitney $U$ test was used to examine the differences in refractive error and choroidal thickness between the AZOOR and unaffected fellow eyes. The Friedman test and Scheffe's paired comparison test were used to examine sequential changes in BCVA, the average threshold on Humphrey perimetry, and the choroidal thicknesses. Spearman's rank correlation coefficient was used to examine a correlation between subfoveal choroidal thickness (SCT) and average threshold. For all tests, $P$ values less than 0.05 were considered statistically significant.

\section{Results}

3.1. Patient Demographics. Table 1 shows the clinical characteristics of AZOOR patients included in this study. The mean age was $40.6 \pm 14.1$ years ranging from 17 to 64 years. The mean follow-up duration was $22.7 \pm 11.3$ 


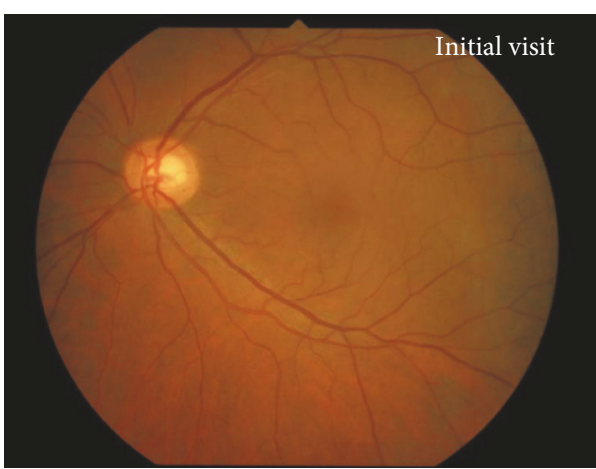

(a)

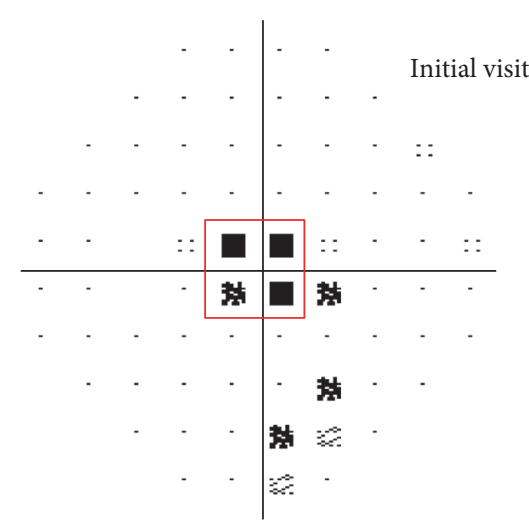

(c)

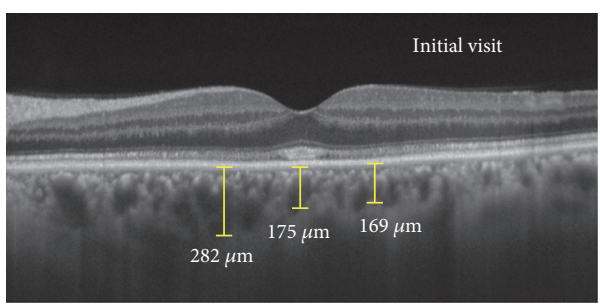

(e)

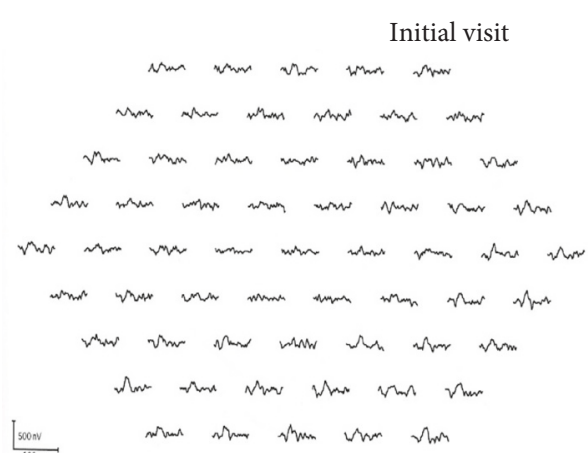

(b)

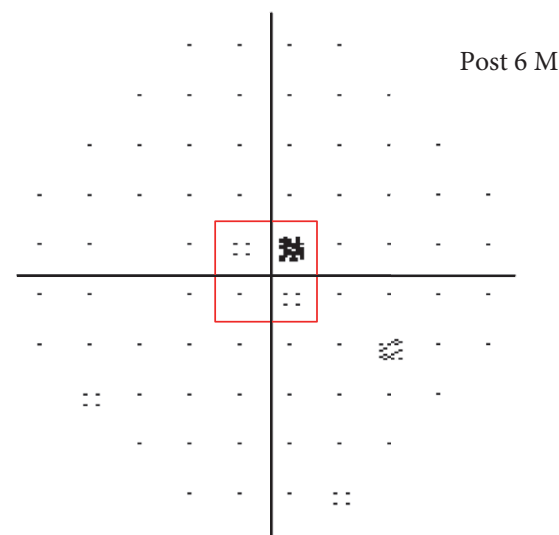

(d)

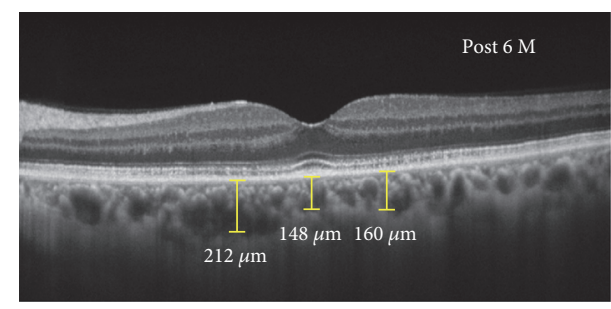

(f)

Figure 1: Photographs of the left eye at the initial visit (a, b, c, and e) and 6 months after the start of systemic corticosteroids therapy (d, f) in a patient (case 9) with acute zonal occult outer retinopathy (AZOOR). (a) Fundus photograph showing a normal retinal appearance. Bestcorrected visual acuity (BCVA) was 1.0. (b) Multifocal electroretinography showing a decreased amplitude at the retinal site corresponding to the visual field defect (Figure 1(c)). (c) Humphrey perimetry showing decreased sensitivity within a central $30^{\circ}$ area. (d) Visual field impairment markedly improved. The BCVA improved to 1.5. (e) Enhanced depth imaging optical coherence tomography (EDI-OCT) revealing the loss of the ellipsoid zone at the macula. Choroidal thicknesses at the subfovea and nasal and temporal sites $1000 \mu \mathrm{m}$ away from the subfovea were 175,282 , and $169 \mu \mathrm{m}$, respectively. (f) The choroidal thicknesses at all sites decreased to 148 , 212 , and $160 \mu \mathrm{m}$, respectively, with the recovery of the macular ellipsoid zone.

months ranging from 6 to 39 months. Six patients had unilateral involvement at the initial visit, and no unaffected fellow eye developed signs of AZOOR during follow-up. The presumed duration from AZOOR onset to the first visit to our hospital was the mean $1.8 \pm 1.8$ months. The mean refractive error was $-4.4 \pm 3.9 \mathrm{D}$ ranging from $-14.25 \mathrm{D}$ to $+0.75 \mathrm{D}$ in the AZOOR eyes $(n=14)$ and $-3.2 \pm 5.0 \mathrm{D}$ ranging from $-14.25 \mathrm{D}$ to $+0.25 \mathrm{D}$ in the unaffected fellow eyes $(n=6)$. AZOOR eyes tended to be more myopic than fellow eyes, but there was no significant difference in refractive error between the two groups $(P=0.34)$.
3.2. Ophthalmic Findings. At the initial visit, the retina was funduscopically normal in 12 of 14 eyes with AZOOR (Figure 1(a), Table 1). The recurrence of AZOOR was not observed in any of the 14 affected eyes within the 6-month follow-up period but in 3 eyes thereafter up to their final visits. None of the 12 eyes with normal retinal appearance at the initial visit demonstrated retinal atrophy thereafter. In 11 eyes examined, single-flash ERG showed a normal amplitude in 7 eyes, reduced a-wave amplitude in 3 eyes, and reduced $b$-wave amplitude in 1 eye. There were noticeably reduced multifocal ERG responses corresponding to visual field loss in all affected eyes (Figure 1(b)). Of the 8 eyes 


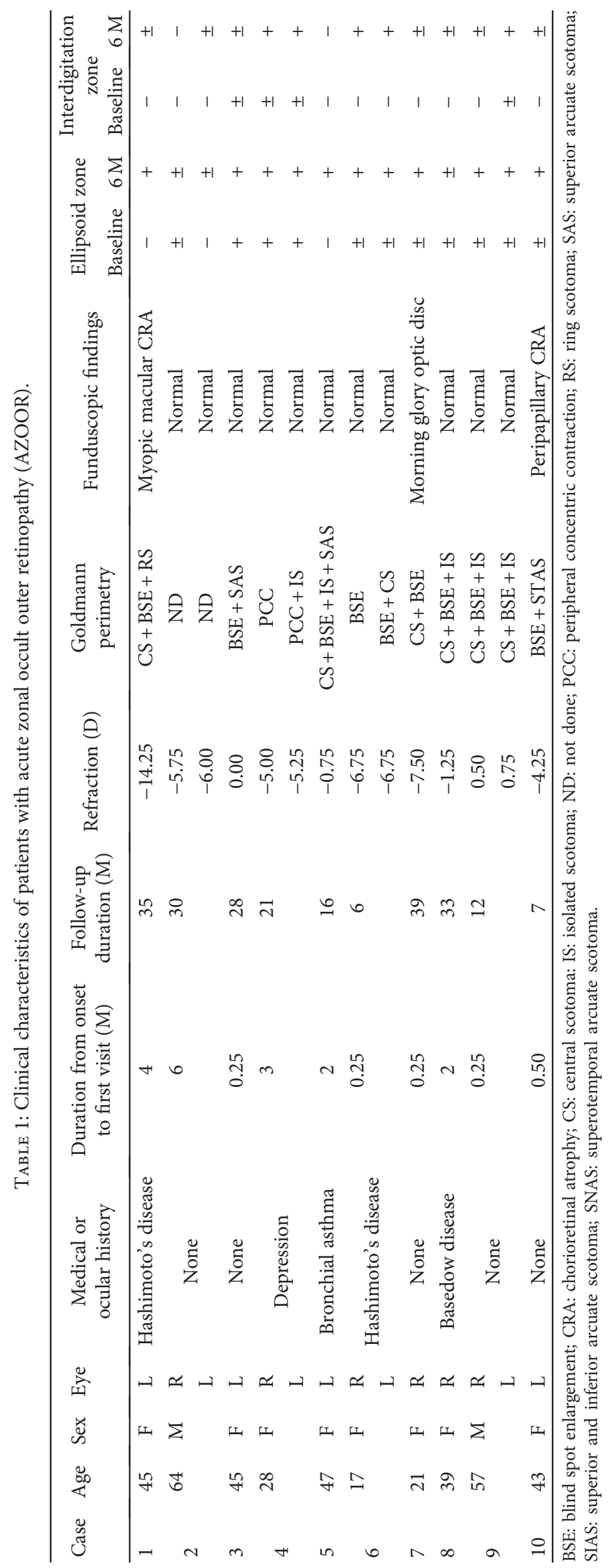




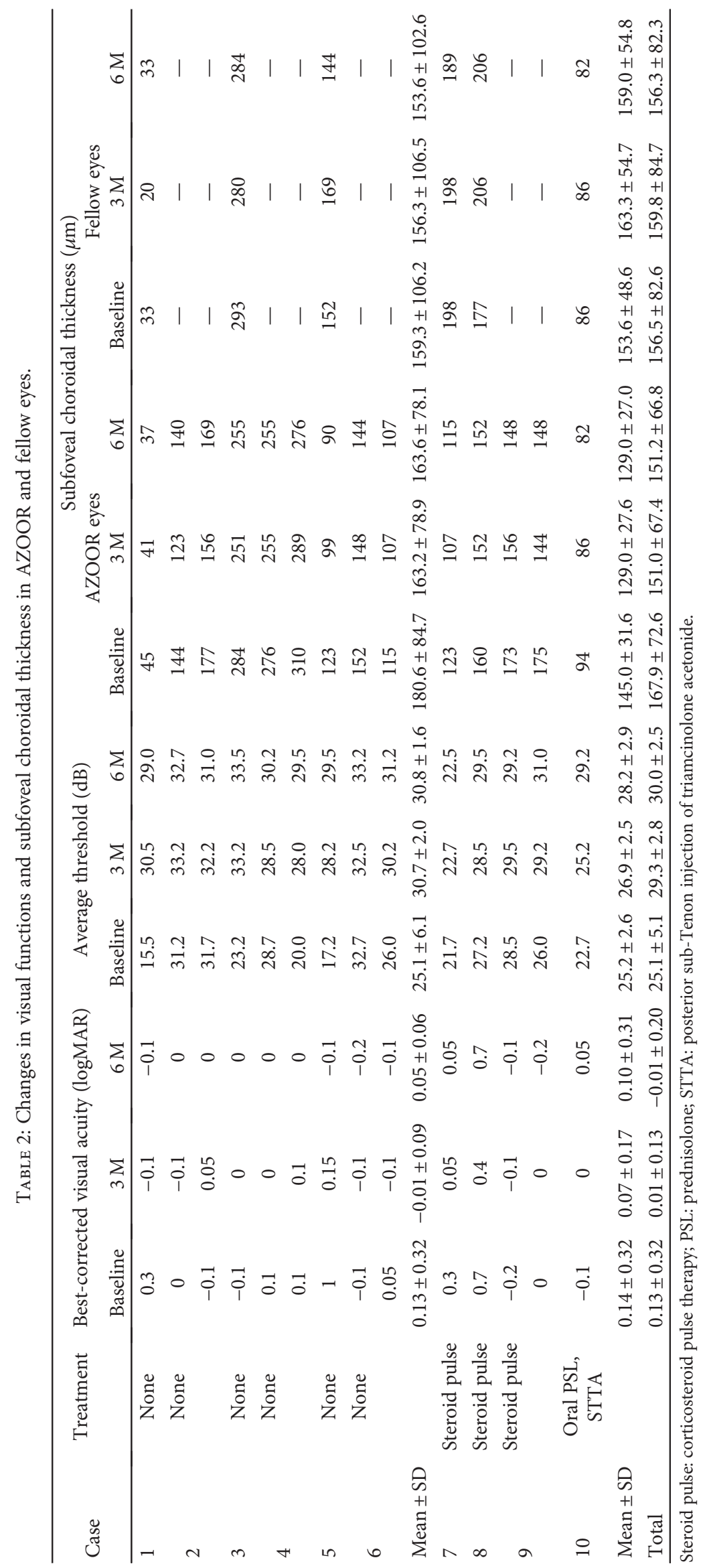




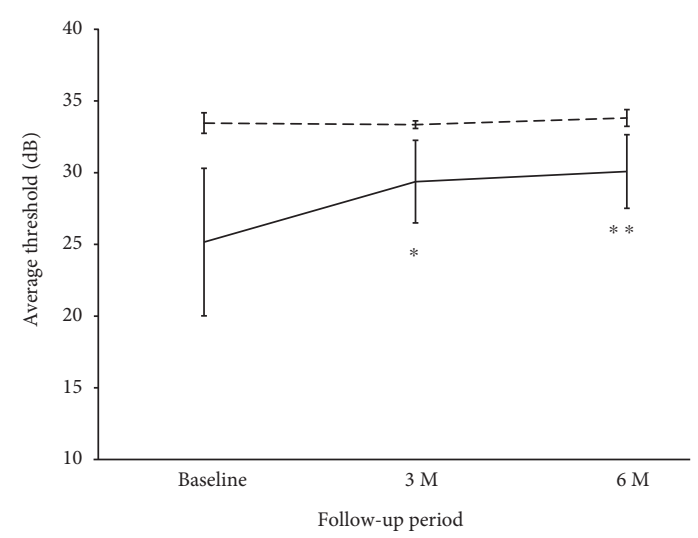

Figure 2: Changes in average threshold on Humphrey perimetry in eyes with AZOOR and unaffected fellow eyes. The "average threshold" is defined as the average values of 4 threshold points surrounded by a red square shown in Figures $1(\mathrm{c})$ and $1(\mathrm{~d})$ at the AZOOR lesion area. In the AZOOR eyes (solid black line), the mean average threshold was significantly higher at 3 and 6 months than at baseline $\left({ }^{*} P<0.05,{ }^{* *} P<0.01\right.$, resp.), whereas it was unaltered in the fellow eyes (dotted line).

examined, fundus autofluorescence showed temporary hyper-autofluorescence in 5 eyes and normal appearance in 3 eyes at the initial visit, corresponding to sites with the visual field impairments.

3.3. Fundus Angiography. FA showed a normal appearance in 10 eyes $(62.5 \%)$, retinal vascular wall staining with leakage in 2 eyes (12.5\%), and optic disc staining in 2 eyes (12.5\%). On ICGA, 4 eyes (28.5\%) appeared normal, but patchy hypofluorescence in the macular area or midperiphery was observed in 7 eyes (50.0\%). In addition, 7 eyes (50.0\%) had diffuse choroidal hyperfluorescence from the posterior pole to the midperipheral region during the middle phase.

3.4. Retinal Morphology. The ellipsoid zone in the AZOORaffected area was invisible in 3 eyes $(21.4 \%)$, discontinuous in 8 eyes $(57.2 \%)$, and normal in 3 eyes $(21.4 \%)$ at the initial visit (Table 1, Figure 1(e)), whereas the interdigitation zone was discontinuous or invisible in all 14 eyes (100\%) at the initial visit. Six months after baseline, the ellipsoid zone was invisible in 0 eyes $(0.0 \%)$, discontinuous in 3 eyes $(21.4 \%)$, and normal in 11 eyes (78.6\%) (Figure $1(\mathrm{f})$ ), while the interdigitation zone was normal in 2 eyes $(14.2 \%)$, discontinuous in 7 eyes $(50.0 \%)$, and invisible in 5 eyes $(35.8 \%)$.

3.5. Changes in Visual Function. Changes in BCVA and the average thresholds at the affected area on Humphrey perimetry in eyes with AZOOR $(n=14)$ are shown in Table 2. In nontreated eyes $(n=9$, cases $1-6)$, the mean $\log$ MAR BCVA showed no significant improvement during the 6-month follow-up period (Friedman's test, $P=0.25)$. In corticosteroid-treated eyes $(n=5$, cases $7-$ $10)$, the BCVA at 3 and 6 months tended to improve compared with the baseline value.

In the AZOOR eyes, the mean average thresholds in the affected area at 3 and 6 months were significantly higher than those at baseline (Figure 2, Friedman's test, $P=0.0008$, and
Scheffe's paired comparison test, $P=0.03$ and $P=0.001$, resp.). Similarly, in the nontreated eyes with AZOOR, the values at 6 months were significantly higher than at baseline (Table 2, Friedman's test, $P=0.03$; Scheffe's paired comparison test, $P=0.03$ ). Also in the corticosteroid-treated eyes, the average threshold showed the same trend. In contrast, the unaffected fellow eyes showed no significant change at 6 months in the average threshold (Figure 2, $P=0.60$ ).

3.6. Changes in Choroidal Thickness. Choroidal thickness changes in the AZOOR eyes and fellow eyes are shown in Tables 2 and 3. In all AZOOR eyes, the SCT values at 6 months were lower than baseline values. The SCT values at 3 and 6 months were significantly lower than baseline (Figure 3(a), Friedman's test, $P<0.0001$; Scheffe's paired comparison test, $P=0.0004, P=0.0002$, resp.). In the unaffected fellow eyes, the mean SCT was unaltered during the 6 month follow-up period (Figure 3(a), $P=0.58$ ). The SCT in the AZOOR eyes was $11.4 \mu \mathrm{m}$ more than in the fellow eyes at baseline, although there was no significant difference between values in two groups $(P=1.00$; Figure $3(\mathrm{a}))$.

Similarly, the choroidal thicknesses at the nasal and temporal sites of the subfovea at 3 and 6 months were significantly lower than baseline values (Table 3, Figures 3(b) and 3 (c), Friedman's test, $P=0.0001, P=0.004$; Scheffe's paired comparison test, $P=0.009$ and $P=0.01, P=0.0001$ and $P=0.01$, resp.). In the unaffected fellow eyes, the thicknesses at the nasal and temporal sites of the subfovea were unaltered during the 6-month follow-up period (Table 3, Figures 3(b) and $3(\mathrm{c}), P=0.58, P=0.21$, resp.).

In the nontreated eyes with AZOOR, values at 3 and 6 months were significantly decreased compared with those at baseline (Figure 3(d), Friedman's test, $P=0.001$; Scheffe's paired comparison test, $P=0.006, P=0.003$, resp.). In the corticosteroid-treated eyes as well, the changes in SCT showed the same trend (Figure 3(d)).

3.7. Correlation between SCT and Visual Function. In the AZOOR eyes, there was a significant inverse correlation between the changing rates of the average threshold and the SCT at 6 months from baseline (Figure $4, R=-0.57$, $P=0.03$ ). No significant difference was found between changes in the SCT and logMAR values of the BCVA during the 6-month follow-up period $(R=0.36, P=0.19)$.

\section{Discussion}

In the present study, by using EDI-OCT during follow-up for eyes with AZOOR, we made the following observations: (1) In both nontreated and corticosteroid-treated AZOOR eyes, the mean average threshold on perimetry in the affected area was significantly increased at 6 months after baseline, with improvement in outer retinal structures, whereas it did not in the unaffected fellow eyes. (2) In the AZOOR eyes, the macular choroidal thicknesses significantly decreased over time compared with baseline, whereas it did not in the unaffected fellow eyes. (3) There was a significant inverse correlation between the changing rates of the average threshold and SCT from baseline to 6 months in the AZOOR eyes. 


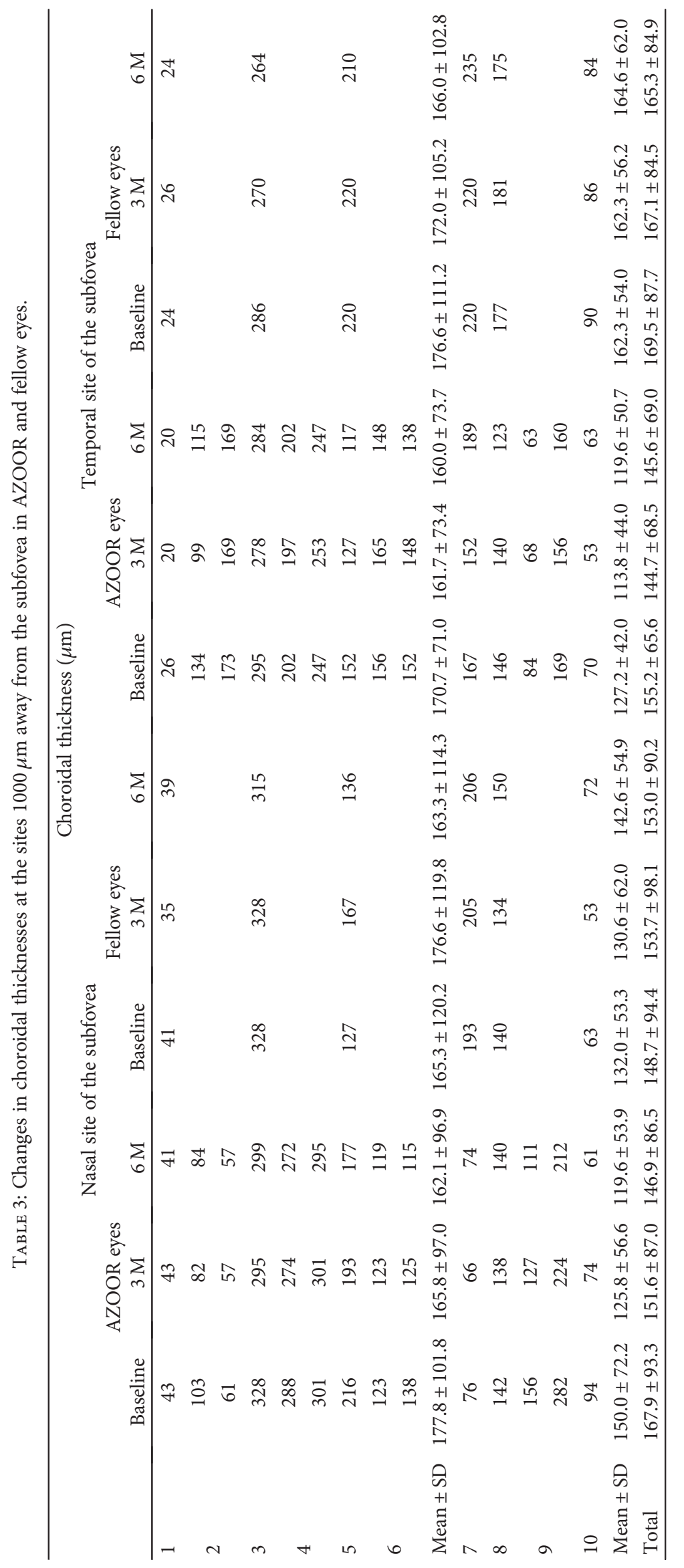




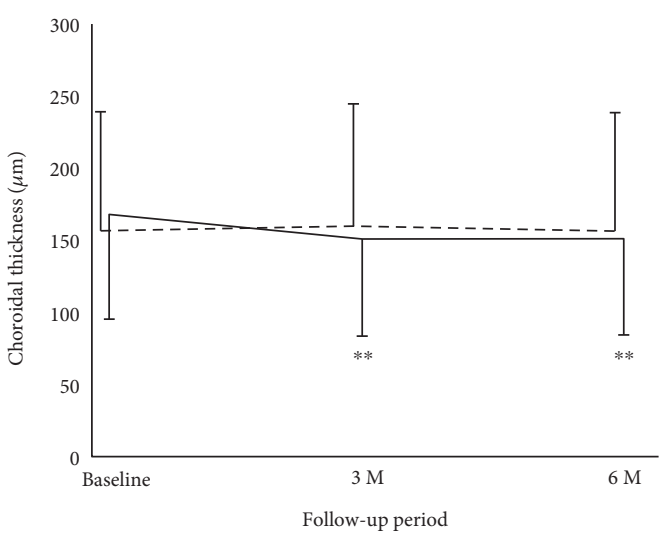

(a)

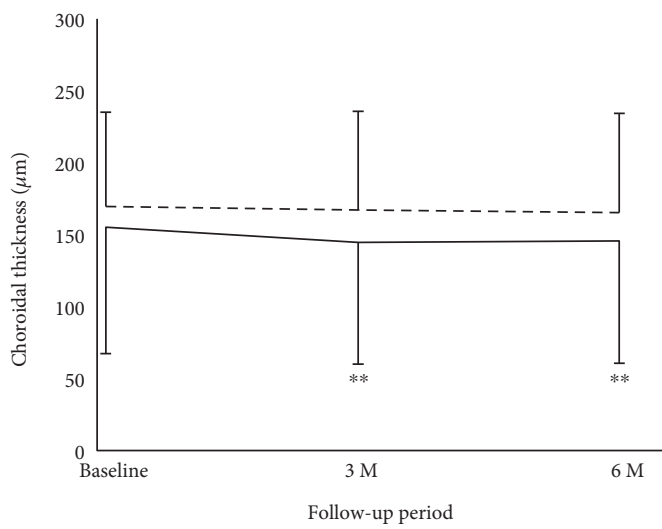

(c)

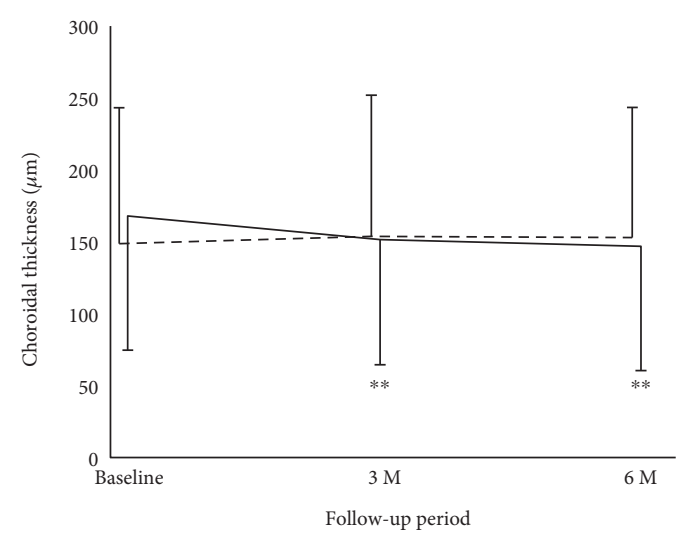

(b)

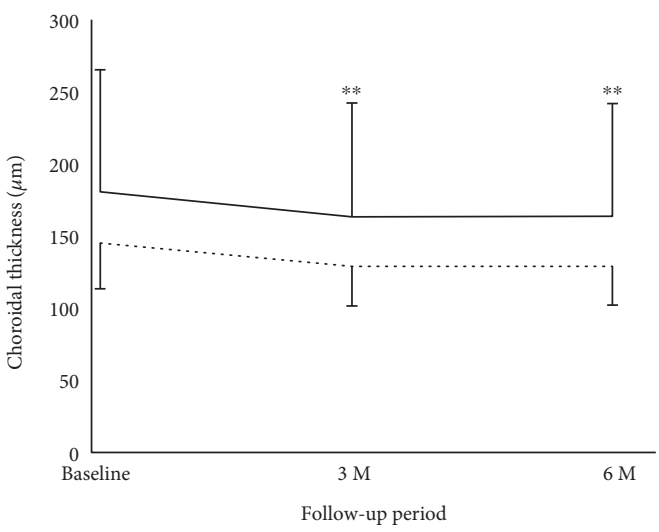

(d)

FIGURE 3: Changes in choroidal thicknesses using EDI-OCT in eyes with AZOOR and unaffected fellow eyes. (a) In the AZOOR eyes (solid black line), the mean subfoveal choroidal thickness (SCT) was significantly reduced at 3 and 6 months compared with baseline $\left(^{* *} P<0.01\right.$, for both), whereas it was unaltered in the unaffected fellow eyes (dotted line). (b) In the AZOOR eyes (solid black line), the mean choroidal thickness at the nasal site $1000 \mu \mathrm{m}$ away from the subfovea was significantly reduced at 3 and 6 months compared with baseline $\left({ }^{* *} P<0.01\right.$, for both), whereas it was unaltered in the unaffected fellow eyes (dotted line). (c) In the AZOOR eyes (solid black line), the mean choroidal thickness at temporal site $1000 \mu \mathrm{m}$ away from the subfovea was significantly reduced at 3 and 6 months compared with baseline $\left({ }^{* *} P<0.01\right.$, for both), whereas it was unaltered in the unaffected fellow eyes (dotted line). (d) Similarly, in the nontreated AZOOR eyes (solid black line), the SCT significantly decreased over time compared with baseline $\left({ }^{* *} P<0.01\right.$, for both). In the corticosteroid-treated eyes, it showed the same trend (dotted line).

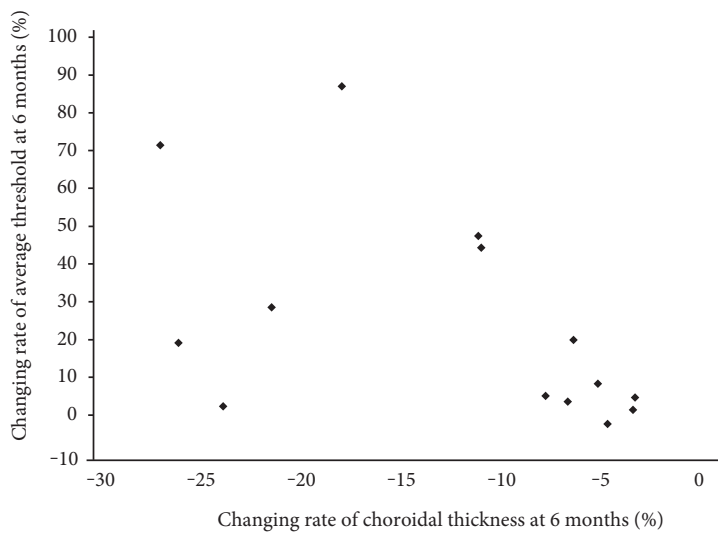

Figure 4: There was a significant inverse correlation between the changing rates of the average threshold and the SCT during the 6 -month follow-up period in AZOOR eyes $(R=-0.57, P=0.03)$.
In the present study, choroidal thickness in AZOOR lesion area significantly decreased with regression of the disease, with a significant correlation between the changes in the visual field impairment and SCT. These results were similar with our previous observations in MEWDS [18]. As concerns the mechanism underlying the sequential reduction in choroidal thickness in AZOOR eyes, two possibilities are speculated as follows: choroidal thinning (permanent volume loss) during the clinical course or transient choroidal thickening at the acute stage. Convalescent tissue loss is theorized to result from atrophic changes due to disease activity, and this is actually the case with some severe cases of AZOOR presenting progressive chorioretinal degeneration over time. However, the present AZOOR eyes in our case series exhibited significant functional improvements in visual field without the development or progression of any funduscopically visible chorioretinal atrophy during the 6-month follow-up period, in accordance with our recent report showing that visual prognosis in Japanese patients with AZOOR was better 
than that in Caucasian patients [12]. Moreover, there were a correlation between the extents of the choroidal thickness reduction and improvement of the visual field defects. These results may not be consistent with the speculation that AZOOR-lesion area in our cases developed choroidal atrophy during follow-up.

In the present study, there were no significant differences in choroidal thicknesses between the AZOOR eyes and the fellow eyes at baseline, although the mean SCT was $11 \mu \mathrm{m}$ more in the AZOOR eyes than in the fellow eyes at baseline. However, the baseline thickness values of the AZOOR eyes might be relatively underestimated, because the degree of myopia tended to be greater in the AZOOR eyes $(-4.4 \mathrm{D})$ than in the control eyes $(-3.2 \mathrm{D})$. Therefore, further studies using healthy subjects with a spherical equivalent matched are needed to examine whether the choroid in AZOOR eyes thickens in the acute stage.

In choroiditis such as Vogt-Koyanagi-Harada disease and serpiginous choroiditis, choroidal thickness decreased and choroidal blood flow velocity increased with regression of these diseases $[19,20]$, consistent with the inflammatory swelling of the choroid with blood flow disruption at the acute stage. These changes in thickness and circulation are therefore called an "inflammatory" pattern in the choroid $[19,20]$ and also seen in chorioretinal diseases with yet unidentified pathogenesis, such as acute posterior multifocal placoid pigment epitheliopathy or unilateral acute idiopathic maculopathy $[21,22]$. Interestingly, this is true with our present (thickness) and previous (circulation) results [8] on AZOOR. The AZOOR complex is a syndrome with outer retinal impairment and includes MEWDS, PIC, acute macular neuroretinopathy (AMN), and multifocal choroiditis and panuveitis other than AZOOR $[1,2]$. It has been reported that choroidal morphologic and circulatory changes in MEWDS, PIC, and AMN showed a similar pattern to AZOOR [18, 23-25]. Taken together, inflammation-related transient choroidal thickening with impaired circulation may be regarded as the common pathogenesis of the AZOOR complex at the active stage.

The primary limitations of our study include its retrospective design and the small sample size. In this study, choroidal thickness was manually measured using EDIOCT B-scan. To overcome this measurement bias, further studies are needed to automatically measure choroidal thickness and volume by using swept-source OCT Cscan. Because of rarity of this disease, both eyes per one patient were used from 4 bilaterally affected cases; however, the statistical significance in SCT changes proved to be still maintained even if only the left eyes from 8 cases (other than cases 7 and 8) were analyzed to avoid any systemic confounders (Friedman's test, $P=0.002$; Scheffe's paired comparison test, $P=0.01, P=0.006$, resp.).

\section{Conclusions}

In AZOOR eyes, choroidal thickness in AZOOR-affected area significantly decreased together with improvements in visual function and outer retinal structure. Furthermore, there was a significant correlation between reduction of choroidal thickness and improvement of visual field defects. These results suggest that choroidal thickness increases at the acute stage of AZOOR. Future studies are needed to further examine the involvement of choroidal thickening in the pathogenesis of AZOOR.

\section{Conflicts of Interest}

All authors have no financial disclosures. The authors declare that there is no conflict of interest regarding the publication of this paper.

\section{References}

[1] J. D. Gass, “Acute zonal occult outer retinopathy. Donders lecture: The Netherlands Ophthalmological Society, Maastricht, Holland, June 19, 1992," Journal of Clinical Neuro-Ophthalmology, vol. 13, no. 2, pp. 79-97, 1992.

[2] J. D. Gass, A. Agarwal, and I. U. Scott, "Acute zonal occult outer retinopathy: a long-term follow-up study," American Journal of Ophthalmology, vol. 134, no. 3, pp. 329-339, 2002.

[3] M. Arai, N. Naoi, A. Sawada, and T. Hayashida, "Multifocal electroretinogram indicates visual field loss in acute zonal occult outer retinopathy," American Journal of Ophthalmology, vol. 126, no. 3, pp. 466-469, 1998.

[4] D. Li and S. Kishi, "Loss of photoreceptor outer segment in acute zonal occult outer retinopathy," Archives of Ophthalmology, vol. 125, no. 9, pp. 1194-1200, 2007.

[5] R. F. Spaide, H. Koizumi, and K. B. Freund, "Photoreceptor outer segment abnormalities as a cause of blind spot enlargement in acute zonal occult outer retinopathy-complex diseases," American Journal of Ophthalmology, vol. 146, no. 1, pp. 111-120, 2008.

[6] T. Fujiwara, Y. Imamura, V. J. Giovinazzo, and R. F. Spaide, "Fundus autofluorescence and optical coherence tomographic findings in acute zonal occult outer retinopathy," Retina, vol. 30, no. 8, pp. 1206-1216, 2010.

[7] S. Mrejen, S. Khan, R. Gallego-Pinazo, L. M. Jampol, and L. A. Yannuzzi, "Acute zonal occult outer retinopathy: a classification based on multimodal imaging," JAMA Ophthalmology, vol. 132, no. 9, pp. 1089-1098, 2014.

[8] M. Saito, W. Saito, Y. Hashimoto et al., "Correlation between decreased choroidal blood flow velocity and the pathogenesis of acute zonal occult outer retinopathy," Clinical \& Experimental Ophthalmology, vol. 42, no. 2, pp. 139-150, 2014.

[9] D. M. Monson and J. R. Smith, "Acute zonal occult outer retinopathy," Survey of Ophthalmology, vol. 56, no. 1, pp. 23-35, 2011.

[10] K. Yasuda, M. Shimura, M. Noro, M. Nakazawa, and M. Tamai, "Clinical course of acute retinal zonal occult outer retinopathy in visual field and multifocal electroretinogram," The British Journal of Ophthalmology, vol. 83, no. 9, pp. 1089-1090, 1999.

[11] A. Saito, W. Saito, N. Furudate, and S. Ohno, "Indocyanine green angiography in a case of punctate inner choroidopathy associated with acute zonal occult outer retinopathy," Japanese Journal of Ophthalmology, vol. 51, no. 4, pp. 295-300, 2007.

[12] S. Saito, W. Saito, M. Saito et al., "Acute zonal occult outer retinopathy in Japanese patients: clinical features, visual function, and factors affecting visual function," PLoS One, vol. 10, no. 4, article e0125133, 2015. 
[13] H. Rodriguez-Coleman, R. G. Bryan, I. Donsoff, R. Nicoletti, and L. A. Yannuzzi, "Zonal occult outer retinopathy," Retina, vol. 22, no. 5, pp. 665-669, 2002.

[14] X. J. Fagan, “A new insight into an old mystery," Clinical \& Experimental Ophthalmology, vol. 42, no. 2, pp. 103-104, 2014.

[15] S. N. Chen and J. F. Hwang, "Ocular coherence tomographic and clinical characteristics in patients of punctuate inner choroidopathy associated with zonal outer retinopathy," Ocular Immunology and Inflammation, vol. 22, no. 4, pp. 263-269, 2014.

[16] X. Zhang, C. Zuo, M. Li, H. Chen, S. Huang, and F. Wen, "Spectral-domain optical coherence tomographic findings at each stage of punctate inner choroidopathy," Ophthalmology, vol. 120, no. 12, pp. 2678-2683, 2013.

[17] K. Tsunoda, K. Fujinami, and Y. Miyake, "Selective abnormality of cone outer segment tip line in acute zonal occult outer retinopathy as observed by spectral-domain optical coherence tomography," Archives of Ophthalmology, vol. 129, no. 8, pp. 1099-1101, 2011.

[18] Y. Hashimoto, W. Saito, M. Saito et al., "Relationship between choroidal thickness and visual impairment in multiple evanescent white dot syndrome," Acta Ophthalmologica, vol. 94, no. 8, pp. e804-e806, 2016.

[19] K. Hirooka, W. Saito, K. Namba et al., "Relationship between choroidal blood flow velocity and choroidal thickness during systemic corticosteroid therapy for Vogt-Koyanagi-Harada disease," Graefe's Archive for Clinical and Experimental Ophthalmology, vol. 253, no. 4, pp. 609-617, 2015.

[20] A. Takahashi, W. Saito, Y. Hashimoto, M. Saito, and S. Ishida, "Impaired circulation in the thickened choroid of a patient with serpiginous choroiditis," Ocular Immunology and Inflammation, vol. 22, no. 5, pp. 409-413, 2014.

[21] K. Hirooka, W. Saito, M. Saito et al., "Increased choroidal blood flow velocity with regression of acute posterior multifocal placoid pigment epitheliopathy," Japanese Journal of Ophthalmology, vol. 60, no. 3, pp. 172-178, 2016.

[22] Y. Hashimoto, W. Saito, M. Saito et al., "Increased choroidal blood flow velocity with regression of unilateral acute idiopathic maculopathy," Japanese Journal of Ophthalmology, vol. 59, no. 4, pp. 252-260, 2015.

[23] K. Hirooka, W. Saito, Y. Hashimoto, M. Saito, and S. Ishida, "Increased macular choroidal blood flow velocity and decreased choroidal thickness with regression of punctate inner choroidopathy," BMC Ophthalmology, vol. 28, no. 14, p. 73, 2014.

[24] K. Hirooka, W. Saito, K. Noda, and S. Ishida, "Enhanceddepth imaging optical coherence tomography and laser speckle flowgraphy in a patient with acute macular neuroretinopathy," Ocular Immunology and Inflammation, vol. 22, no. 6, pp. 485489, 2014.

[25] Y. Hashimoto, W. Saito, M. Saito et al., "Decreased choroidal blood flow velocity in the pathogenesis of multiple evanescent white dot syndrome," Graefe's Archive for Clinical and Experimental Ophthalmology, vol. 253, no. 9, pp. 1457-1464, 2015. 


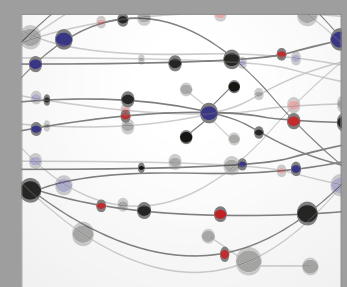

The Scientific World Journal
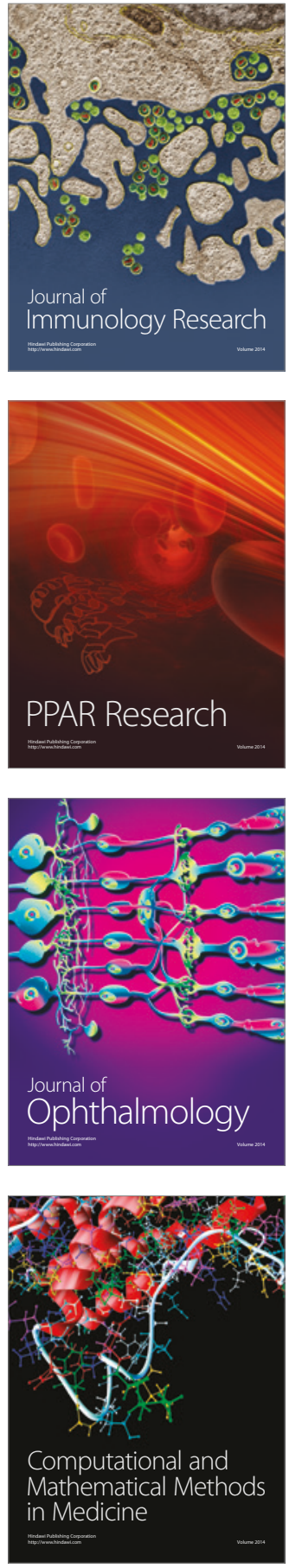

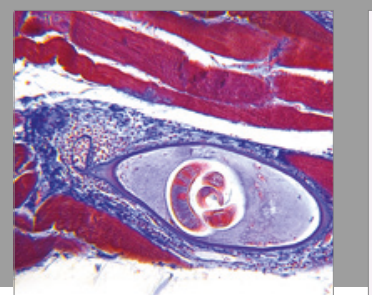

Gastroenterology Research and Practice
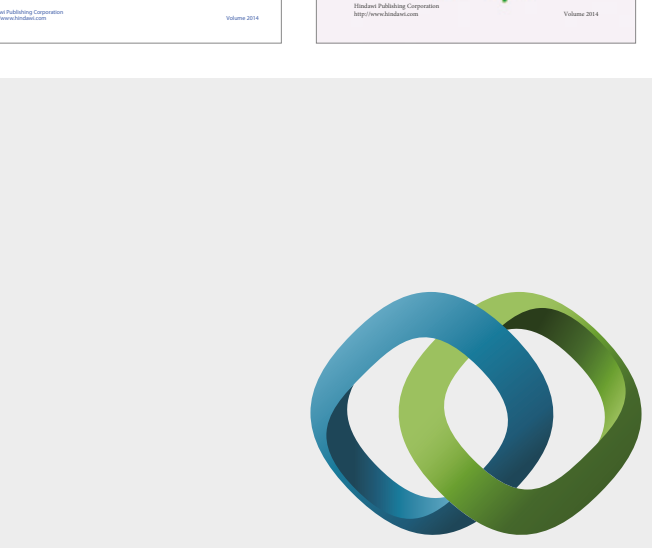

\section{Hindawi}

Submit your manuscripts at

https://www.hindawi.com
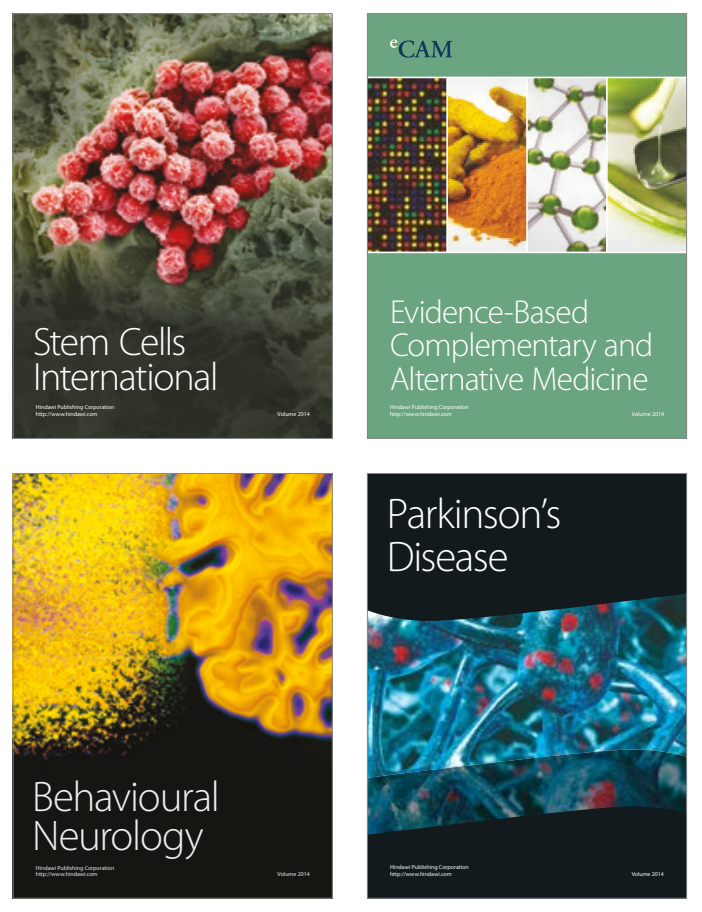
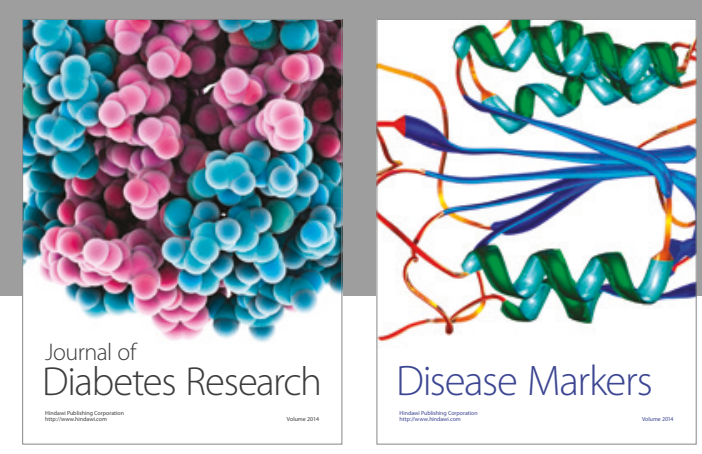

Disease Markers
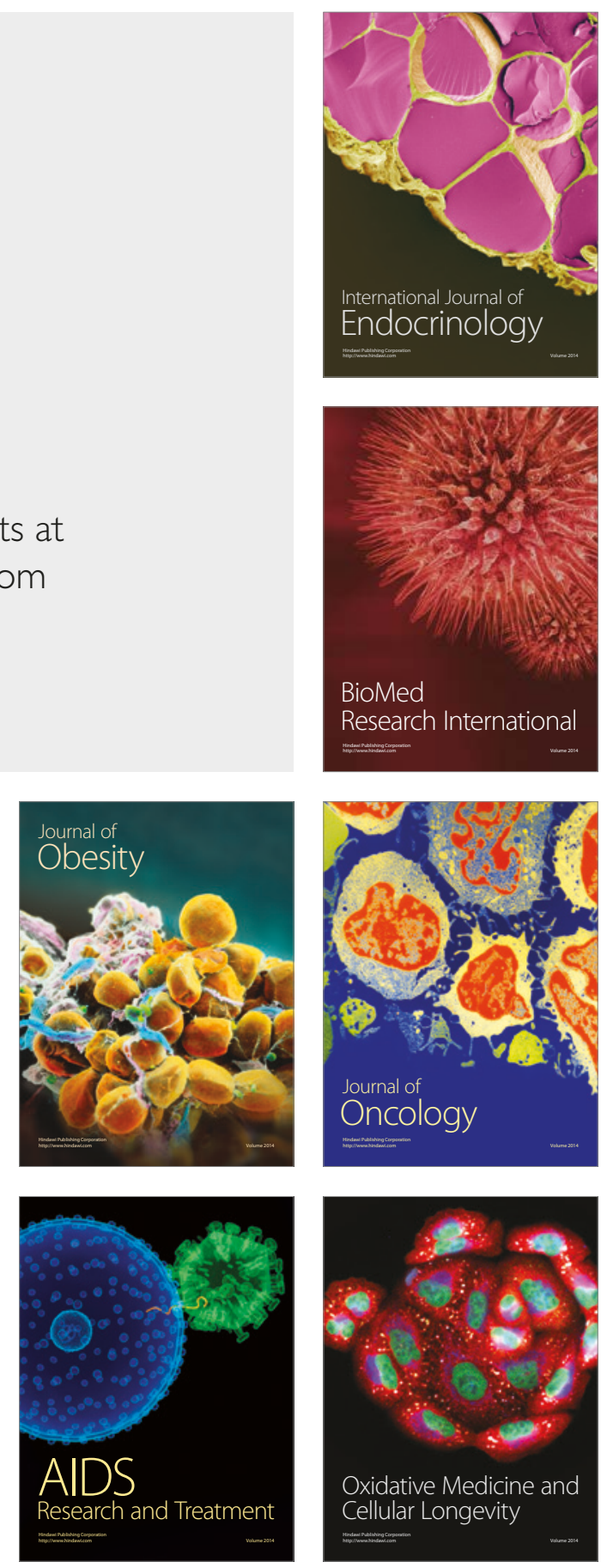